\title{
Reason and emotion in advocacy
}

Am. Sociol. Rev. https://doi.org/10.1177/000312241

7733673 (2017)

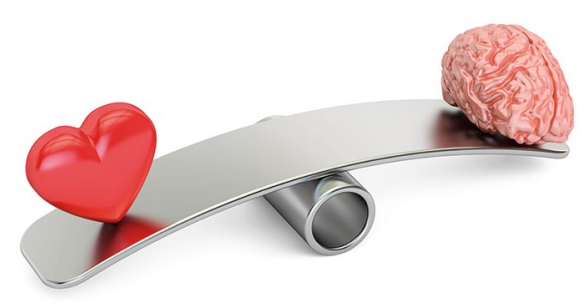

Credit: Olekcii Mach / Alamy Stock Photo

To raise awareness about their causes and ultimately shift public attitudes, advocacy organizations need to stimulate public conversation. Different theories emphasize either rational arguments or emotional appeals as the most successful means through which advocacy organizations can draw people into conversation.

New research by Christopher Bail, Taylor Brown and Marcus Mann at Duke University comes to a different conclusion: organizations can best engage the public in conversation if they produce rational arguments after extended emotional deliberation, and vice versa. The authors used text-analysis methods to measure the frequency of emotional and cognitive language use within two advocacy fields, autism spectrum disorders and human organ donation, on Facebook over a period of 1.5 years. They then applied time-series models to the data, finding evidence for 'cognitive-emotional currents': an inverse relationship between cognitive and emotional language use over time. Using data collected from 92 relevant advocacy organizations over the same period, the authors show that organizations whose posts contribute to 'phase shifts' in the conversation (from emotional to rational debate and vice versa) are more likely to stimulate conversation.

This work furthers our understanding of the nature of public deliberation and offers valuable insight for advocacy organizations, which face considerable competition for public attention.

Stavroula Kousta

Published online: 2 November 2017

https://doi.org/10.1038/s41562-017-0254-7 